\title{
Effect of Methanol Extract from Cassia mimosoides var. nomame on Ischemia/Reperfusion-induced Renal Injury in Rats.
}

\author{
Hae Sook Baek ${ }^{1 \#}$, Sun Ha Lim, Ki Sung Ahn², Jong Won Lee ${ }^{1 *}$ \\ 1 : Department of Biochemistry, School of Medicine, Catholic University of Daegu, Daegu 705-718, Korea \\ 2 : Department of Internal Medicine, School of Medicine, Catholic University of Daegu, Daegu 705-718, Korea
}

\begin{abstract}
Objectives : The purpose of this study was to determine whether the methanol extract of Cassia mimosoides var. nomame Makino, a naturally growing plant in Korea, could prevent the renal-ischemia/reperfusion injury in a rat model or not.

Methods : The radical scavenging activities of the extracts, and ascorbic acid as a positive control, were measured in vitro. At one hour after an intraperitoneal injection of the extract $(400 \mathrm{mg} / \mathrm{kg}$ ), renal ischemia/reperfusion injury was generated by $40 \mathrm{~min}$ clamping of the left renal artery in rats. After renal ischemia/reperfusion and $24 \mathrm{hr}$ restoration of blood circulation, the serum creatinine concentration was measured. And the extent of epithelial cell injury and apoptosis was assessed by various staining technologies. The Bax/Bcl-2 ratio and activated caspase- 3 were assessed by immunohistochemistry.

Results : The extract showed a slightly lower level of radical scavenging activity than that of ascorbic acid. Compared to those of the vehicle-treated group, the extract-treated group displayed a significantly smaller tubular epithelial cell injury of 54\% reduction in the outer medulla region and a lower serum creatinine concentration of $50 \%$ reduction. It seems that the reduction in cellular injury is due to the attenuation of the Bax/Bcl-2 ratio, and the inhibition of caspase-3 activation by the extract of Cassia mimosoides.

Conclusions : Cassia mimosoides var. nomame Makino could be a good candidate for a prophylactic agent against the ischemia/reperfusion-induced kidney injury.
\end{abstract}

Key words : Cassia mimosoides var. nomame Makino, methanol extract, kidney, ischemia/reperfusion, reactive oxygen species, apoptosis

\section{Introduction}

Ischemic acute renal failure a subset of acute renal failure (ARF), also known as acute kidney injury ${ }^{1,2)}$, is attributed to rise in the creatinine concentration resulting from a reduction of blood flow to the kidneys $^{3,4)}$. In the severe cases of the event such as sepsis or cardiac surgery, interruption or prolonged reduction of blood flow results in ischemia to the kidney, especially in the outer medulla region. The condition is caused by a combination of countercurrent oxygen exchange and selective reduction in blood supply ${ }^{5}$, which subsequently injures the tubular cells by depleting $\mathrm{ATP}^{3,6)}$. Although early restoration of the blood flow to ischemic kidneys is one way to prevent ischemic $\mathrm{ARF}$, the method predisposes the already-weakened cells to further injuries by augmenting the production of reactive oxygen species $(\mathrm{ROS})^{7,8)}$. The sequence of ischemia followed by reperfusion (ischemia/reperfusion) can destroy the tubular cells by forcing them to undergo apoptosis or necrosis ${ }^{9)}$. Thus, one way to prevent ischemic ARF caused by ischemia/reperfusion injury is to attenuate tubular cell death by eliminating ROS and consequently inhibiting apoptosis ${ }^{6,10)}$.

Cassia mimosoides var. nomame Makino (CM) grows

\footnotetext{
*Corresponding Author : Jongwon Lee. Department of Biochemistry School of Medicine Catholic University of Daegu Daegu 705-718 Korea. ·Tel : 82-53-650-4471 ·Fax : 82-53-621-9206 ·E-mail : leejw@cu.ac.kr

\#First Author : Hae Sook Baek. Department of Biochemistry School of Medicine Catholic University of Daegu Daegu 705-718 Korea

·Tel : 82-53-650-4499 · Fax : 82-53-621-9206 ·E-mail : sftwtmt@hanmail.net

·접수 : 2013년 10월 23일 · 수정 : 2013년 11월 07일 · 채택 : 2013년 11월 08일
} 
naturally in Korea as well as in China and Japan. Historically, the whole plant of which has been used as a source of food, especially as an ingredient for tea $^{11,12)}$. In addition, extracts of CM, their fractions and components isolated have various physiological activities $^{11,13)}$. For example, a fraction obtained by ethanol extraction and by subsequent column chromatography ${ }^{11}$, and flavan dimers purified from $\mathrm{CM}^{13)}$ have lipase inhibition activities and anti-obesity effects.

In our previous study, we reported that a methanol extract of CM reduced brain and myocardial injury in a rat model of ischemia/reperfusion when the extract was administered by an intraperitoneal injection prior to occlusion ${ }^{14,15)}$. Since the underlying mechanisms for ischemia/reperfusion injury to kidney, heart, and brain are similar ${ }^{16)}$, CM was hypothesized to be effective in preventing ischemic ARF as well. In this study, the validity of the hypothesis was tested with a focus on the effectiveness of $\mathrm{CM}$ in ablating ROS and, consequently, attenuating apoptosis.

\section{Materials and Methods}

\section{Extraction}

A methanol extract of the whole plant of $\mathrm{CM}$ was prepared as previously described ${ }^{10,14,15)}$. Briefly, the CM specimens collected from Uiseong area, a county of Gyeongsangbuk-do, South Korea, were washed, and dried [A voucher specimen of CM was deposited in Nutrition Biochemistry Laboratory, Catholic University of Daegu, Daegu, Korea]. Then, $500 \mathrm{~g}$ of the dried sample was sonicated using an ultrasonicator (8210R-DTH, Branson Ultrasonic Corp., Danbury, CT), extracted in $5 \mathrm{~L}$ methanol twice for 24 $\mathrm{hr}$ each at room temperature, and filtered with filter papers (Whatman No. 3, Whatman Inc., Piscataway, NJ). The filtrate was vacuum-dried using a rotary evaporator [NP-1, Tokyo Rikakikai Co. (EYELA), Tokyo, Japan] to yield a 62 g extract.

\section{Assessment of radical scavenging activity}

\section{of extract with DPPH}

Various amounts of the extract dissolved in $25 \mu \mathrm{L}$ of dimethyl sulfoxide (DMSO) were mixed with $2.5 \mathrm{~mL}$ of reaction solution containing $\alpha, \alpha$-diphenyl- $\beta$ -picrylhydrazyl (DPPH) [0.1 mM DPPH, 40\% ethanol and $0.04 \mathrm{M}$ acetate buffer $(\mathrm{pH} 5.5)]$. Thirty minutes after incubation of the samples at $37{ }^{\circ} \mathrm{C}$, the absorbance was measured at $517 \mathrm{~nm}$ with a UV-visible spectrophotometer (U2800, Hitachi, Japan).
The experiment was repeated three times, and the average was recorded. The final concentrations of the extract in the samples were 0 (negative control), 10, 50, 100, and $200 \mu \mathrm{g} / \mathrm{ml}$. Ascorbic acid samples diluted to the same concentrations as the extract samples were used as positive controls. Radical scavenging activity of the extract and ascorbic acid represented by electron donating activity (EDA) was calculated with the following equation

$\operatorname{EDA}(\%)=(1-$ extract or ascorbic absorbance/blank absorbance) x 100

in which the terms extract, ascorbic acid, and blank absorbance represent the absorbance of solutions containing the extract, ascorbic acid, and nothing (negative control), respectively ${ }^{17,18)}$.

\section{Animals}

Eight-week-old male Sprague Dawley (SD) rats were purchased from Samtaco Inc. (Osan, Korea). Experiments were carried out according to the guidelines for animal care and protocols for the use of laboratory animals were approved by the Institutional Animal Care and Research Advisory Committee of Catholic University of Daegu. Animals were housed in a temperature-controlled environment under diurnal lighting conditions with food and water available ad libitum until the day of the experiment.

\section{Rat renal ischemia/reperfusion model}

A rat renal ischemia/reperfusion model was prepared as described previously ${ }^{10)}$. Briefly, renal ischemia/reperfusion injury was generated in male SD rats (250-300 g) by surgically removing their right kidneys and then clamping their left renal arteries as previously described ${ }^{15)}$. Briefly, the rats were anesthetized with intraperitoneal injections of ketamine (100 $\mathrm{mg} / \mathrm{kg}$ ) and xylazine $(5 \mathrm{mg} / \mathrm{kg})$. Their body temperatures were maintained at $36 \pm 0.5{ }^{\circ} \mathrm{C}$ throughout the experiments, with a heating pad. After midline incision, their right kidneys were surgically removed. Then, the remaining left kidneys underwent renal artery occlusion by clamping for $40 \mathrm{~min}$ (ischemia) followed by recirculation of blood for $24 \mathrm{hr}$ (reperfusion), after which the rats were sacrificed for further examinations.

\section{Extract administration}

The rats were randomly assigned to one of the three groups: (1) extract-treated group (I/R + extract) $(\mathrm{n}=10)$, (2) vehicle-treated group $(\mathrm{I} / \mathrm{R})(\mathrm{n}=$ 
10), or (3) sham group $(n=7)$. In the extract-treated group, the rats received the extract $(400 \mathrm{mg} / \mathrm{kg})$ dissolved in $0.3 \%$ carboxymethyl cellulose by an intraperitoneal injection $1 \mathrm{hr}$ prior to the occlusion. In the vehicle-treated group, the rats received only the $0.3 \%$ carboxymethyl cellulose by an intraperitoneal injection $1 \mathrm{hr}$ prior to the occlusion. In the sham group, the experimental procedure was the same as in the vehicle-treated group except that there was no occlusion by clamping.

\section{Serum creatinine levels}

Serum creatinine levels were measured as described previously ${ }^{10)}$. Briefly, at the end of the reperfusion period $(24 \mathrm{hr}$ ), blood samples were collected from the renal veins while the rats were anesthetized under ketamine and xylazine. The serum creatinine concentration was measured by the Jaffe reaction on a Hitachi 747 analyzer (Roche Diagnostics, Germany).

\section{Histological analysis}

The remaining left kidney was harvested, bisected along its long axis, fixed in 10\% neutral formalin solution for 24-28 hr, embedded in paraffin, and sectioned on slides at $5-\mu \mathrm{m}$ thickness. The sections were deparaffinized as described previously ${ }^{10)}$ and stained with hematoxylin and eosin (H\&E). Twenty representative photomicrographs were randomly taken in the outer medulla region of one representative section at 200x magnification under a light microscope. The percentage of the damaged area in the section was assessed by computer-aided image analysis (ImageJ) from which the degree of damage was graded on a 5 -point scale (0-4) adopted by Kelly et al. ${ }^{19)}$, with a slight modification in assigning the range of scores: 0, none; 1, 〈 5\%; 2, 5-30\%; 3, 30-50\%; 4, >50\%. In a blind-test, the H\&E-stained sections were prepared by the first author (HSB), and the grading was performed by the second author (SHL). Scores of the 20 sections were averaged to represent a score for each rat. The number of rats assessed for the histological examination was as follows: (1) extract-treated group $(\mathrm{n}=7)$, (2) vehicle-treated group group (I/R) ( $=7)$, and (3) sham group $(\mathrm{n}=5)$.

\section{TUNEL staining}

To measure DNA nicks, terminal deoxynucleotidyltransferase-mediated dUTP nick-end labeling (TUNEL) staining was performed using ApopTag $^{\circledR}$ in situ apoptosis detection kit (Chemicon,
Temecula, CA) according to the manufacturer's protocol; that is, after successive pretreatments with Proteinase $\mathrm{K}$ and hydrogen peroxide, the sections were incubated in terminal deoxynucleotidyl transferase (TdT) and dNTP covalently linked with digoxigenin for $30 \mathrm{~min}$ at $37{ }^{\circ} \mathrm{C}$. Then, they were incubated in anti-digoxigenin peroxidase conjugate, and finally 3,3'-diaminobenzidine tetrahydrochloride (DAB), which is a substrate for staining. For negative controls, TUNEL staining was performed in the absence of TdT. For the counter-staining, the sections were stained with methyl green. To determine the percentage of apoptotic cells, micrographs of TUNEL-positive nuclei and methyl green-stained nuclei were captured using an Olympus microscope. The cell number was counted using the ImageJ software (ImageJ version 1.43r; NIH) from 20 random fields at $400 \times$ magnification. In a blind test, the TUNEL-stained sections were prepared by the first author (HSB), and the percentage of apoptotic cells was assessed by the second author (SHL).

\section{Assessment of Bcl-2, Bax, and caspase -3}

To assess the presence of Bcl-2, Bax, and cleaved (activated) caspase-3, immunohistochemical techniques were utilized as described previously ${ }^{10)}$. Briefly, the sections were incubated overnight at $4{ }^{\circ} \mathrm{C}$ in a humidified chamber immersed in a $0.1 \%$ BSA solution containing one of the following antibodies: rabbit polyclonal anti-Bcl-2, anti-Bax (1:50 dilution, Santa Cruz Biotechnology Inc., Santa Cruz, CA) or anti-(cleaved caspase-3) primary antibody (1:50 dilution, Cell Signaling, Beverly, MA). A Vectastain Elite ABC kit (Vector Laboratories, Burlington, ON) was used for staining, which was carried out according to the manufacturer's instructions; specifically, the sections were incubated for $5 \mathrm{~min}$ in PBS containing $1 \mathrm{mg} / \mathrm{mL}$ 3,3'-diaminobenzidine tetrahydrochloride (DAB) and $0.3 \% \mathrm{H}_{2} \mathrm{O}_{2}$ for color development. Then, they were evaluated at 200x magnification. The color intensity of DAB staining was measured using ImageJ software. To allow for a quantitative comparison, the color intensity value for the sham group was arbitrarily set as 1.0. In a blind test, the $\mathrm{DAB}$-stained sections were prepared by the first author (HSB), and the color intensity was evaluated by the second author (SHL).

\section{Statistical analysis}

The data were recorded as the means \pm SEM. The groups were compared following the standard statistical methods using the SPSS software (IBM SPSS Statistics; version 19, Armonk, NY, USA). The data were 
analyzed by one-way ANOVA, Kruskal-Wallis one-way ANOVA on ranks, or unpaired t-test. The statistical significance was determined at $P<0.05$ level.

\section{Results}

\section{Radical scavenging activity of the extract}

To determine whether the extract had an antioxidant property, its radical scavenging activity was assessed by measuring the degree to which it could quench $\mathrm{DPPH}$. As the concentrations of the extract increased, the absorbance of the samples at $517 \mathrm{~nm}$ decreased (Fig. 1A). A similar trend was observed for ascorbic acid which is a strong antioxidant. These findings can be attributed to the fact that the electron donating ability (EDA) (\%) of the extract and ascorbic acid increases proportionally with their concentrations (Fig. 1B). IC $50 \mathrm{~S}$, the concentrations at half-maximal EDA, of the extract and ascorbic acid were 43.4 and 24.5 $\mu \mathrm{g} / \mathrm{mL}$, respectively.

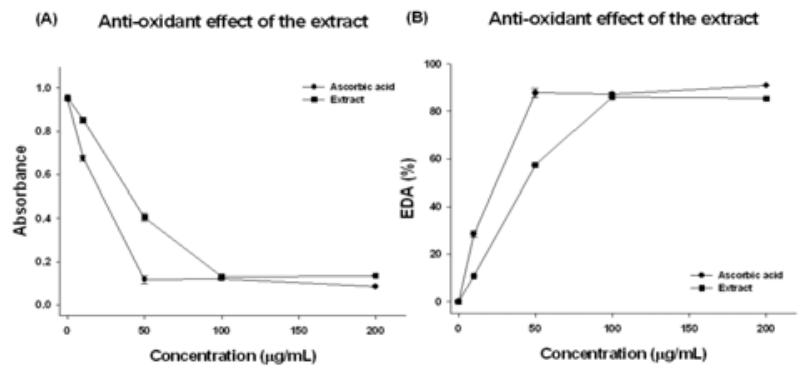

Fig. 1. Assessment of DPPH radical scavenging activity of methanol extract. Absorbance at $517 \mathrm{~nm}$ was measured after various concentrations of the extract and ascorbic acid were reacted with DPPH (A), and then electron donating ability (EDA) of the methanol extract of Cassia mimosoides var. nomame Makino and ascorbic acid was calculated using the following equation: $\mathrm{EDA}(\%)=(1$ - extract or ascorbic acid absorbance/blank absorbance) $\times 100$ (B), in which the terms extract, ascorbic acid, and blank absorbance represent the absorbance of solutions containing the extract, ascorbic acid, and nothing (negative control), respectively.

\section{Improvement of renal function and}

\section{attenuation of tubular damage by the extract}

To determine whether the extract preserved the renal function amidst the ischemia/reperfusion injury, serum creatinine level was selected as a biomarker. In the rat model of ischemia/reperfusion, the rats underwent removal of the right kidneys and subsequent occlusion of the remaining left kidneys by a 40-min clamping to induce ischemia, followed by a $24-\mathrm{hr}$ reperfusion. The extract $(400 \mathrm{mg} / \mathrm{kg})$ was administered by an intraperitoneal injection $1 \mathrm{hr}$ prior to the occlusion of the remaining left kidney. Administration of the extract did affect adversely to the rats, including mortality. The serum creatinine levels of the extract-treated group were much lower compared to those of the vehicle-treated group $(1.1 \pm 0.9$ versus $2.3 \pm 1.2 \mathrm{mg} / \mathrm{dL}$, $P<0.05)$ (Fig. 2A), whereas those of the sham group remained low $(0.3 \pm 0.1 \mathrm{mg} / \mathrm{dL})$. To determine whether low serum creatinine level was correlated with protection of tubular cells in outer medulla regions against ischemia/reperfusion injury, the sections were stained with H\&E and analyzed (Fig. 2B, C). Representative photomicrographs revealed that the tubules in the vehicle-treated group were damaged as shown by their distinctive morphological changes, loss of nuclei and concomitant death of tubular epithelial cells, and desquamation of viable and necrotic cells, which became apparent when compared to those in the sham group (Fig. 2B). The extent and severity of tubular damage in the extract-treated group were much less, compared to those in the vehicle-treated group. To quantify the extent of damage, a grading system on a 5 -point scale (0-4) was adopted as described in the Materials and Method section (Fig. 2C). The result was consistent with the qualitative findings; tubular damage in the extract-treated group $(n=7)$ was much less, compared to that in the vehicle-treated group $(\mathrm{n}=7)$ (average score $1.6 \pm 0.2$ versus $3.5 \pm 0.3, P<$ $0.05)$, where "score 0 " represents no damage as was confirmed in the sham group $(n=5)$. 
(A)

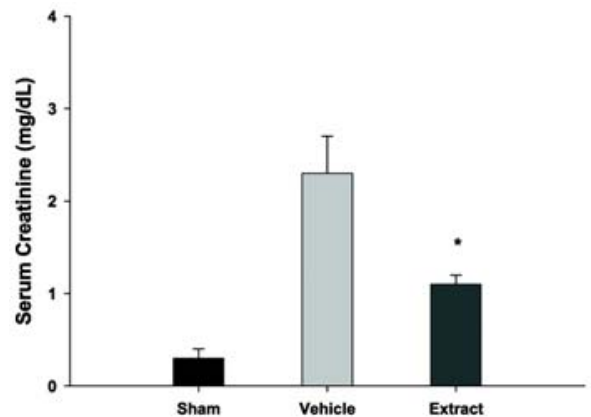

(B)

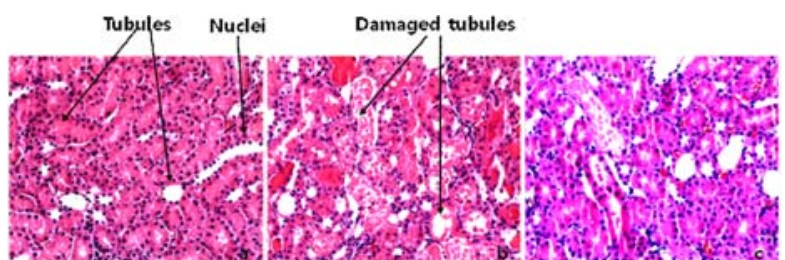

(C)

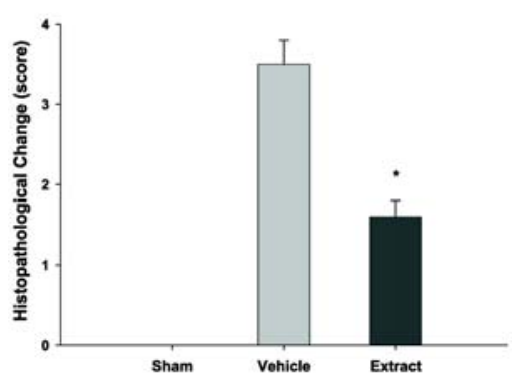

Fig. 2 Measurement of serum creatinine levels and histopathological evaluation of tubular injury in a rat model of ischemia/reperfusion injury. A. Serum creatinine levels in the sham $(n=7)$, vehicle-treated $(\mathrm{l} / \mathrm{R})(\mathrm{n}=10)$ and extract-treated $(\mathrm{l} / \mathrm{R}+400 \mathrm{mg} / \mathrm{kg}$ extract $)(\mathrm{n}=10)$ group were measured after renal ischemia/reperfusion injury. I/R injury was generated by $40 \mathrm{~min}$ clamping of the left renal artery in Sprauge-Dawley rats after surgical removal of the right kidney, followed by $24 \mathrm{hr}$ restoration of blood circulation. In the extract-treated group, the rats received the extract $(400 \mathrm{mg} / \mathrm{kg}$ ) dissolved in $0.3 \%$ carboxymethyl cellulose (CMC) by an intraperitoneal injection $1 \mathrm{hr}$ prior to occlusion. In the vehicle-treated group, the rats received only the $0.3 \% \mathrm{CMC}$ by an intraperitoneal injection 1 $\mathrm{hr}$ prior to the occlusion. In the sham group, experimental procedure was the same as in the vehicle-treated group except that there was no occlusion by clamping. The numbers of the rats used in the sham, vehicle-treated, and extract-treated group were 7,10 , and 10, respectively. Each column represents the means \pm SEM ${ }^{*} P$ < 0.05 vs. control group. B. Representative photomicrographs of the renal tissue sections stained with haematoxylin \& eosin (H\&E) at magnification $200 \times$ were presented: (a) sham $(n=5)$, (b) vehicle-treated $(n=7)$, and (c) extract-treated group $(n=7)$, respectively. C. Semi-quantitative assessment with $\mathrm{H} \& \mathrm{E}$ of the histological alterations due to tubular damage was presented on a 5 -point scale system: 0, none; 1, < $5 \%$; 2, 5-30\%; 3, 30-50\%; 4, >50\%. Each column represents the means \pm SEM. ${ }^{*} P<0.05$ vs. vehicle-treated group.

\section{Involvement of apoptosis in the attenuation}

\section{of tubule damage by the extract}

Levels of DNA nicks and cleaved caspase-3 measured by TUNEL staining and immunohistochemistry, respectively, were selected as indices to assess the extent of apoptosis that occurred in the tubular epithelial cells during ischemia/reperfusion (Fig. 3A, B). Representative photomicrographs stained with TUNEL revealed apoptotic cells in the tubules of the sham, vehicle-treated and extract-treated group (Fig. 3A). The number of apoptotic cells in the extract-treated group and the sham group was much lower than that in the vehicle-treated group. To quantify these findings, the ratio of apoptotic cells to total cells, [Apoptotic cell (\% total cell)], was assessed (Fig. 3B). The ratio was significantly lower in the extract-treated group $(n=7)$ compared to that in the vehicle-treated group $(\mathrm{n}=7)$ $(13.3 \pm 1.3$ versus $36.0 \pm 8.4 \%, P<0.05)$, whereas the ratio in the sham group $(n=5)$ remained low $(0.7 \pm 0.1$ $\%)$. Used as another index for assessing apoptosis, representative micrographs stained with an antibody that specifically recognizes cleaved caspase-3 (activated caspase-3) also revealed apoptotic cells in the tubules of the sham, vehicle-treated and extract-treated group (Fig. 3C, D). The result was consistent with the findings observed via TUNEL staining; the color intensity, which reflects the number of apoptotic cells, of the extract-treated group and the sham group was markedly less than that of the vehicle-treated group (Fig. 3C). To quantify these findings, the color intensity in each group was compared to each other (Fig. 3D). The color intensity of the extract-treated group $(n=7)$ was much lower than that of the vehicle-treated group $(n=7) \quad(5.6 \pm 0.4$ versus $10.2 \pm 0.3$ $\%, \mathrm{P}<0.05)$, when the intensity of the sham group was arbitrarily set as 1.0 . 
(A)

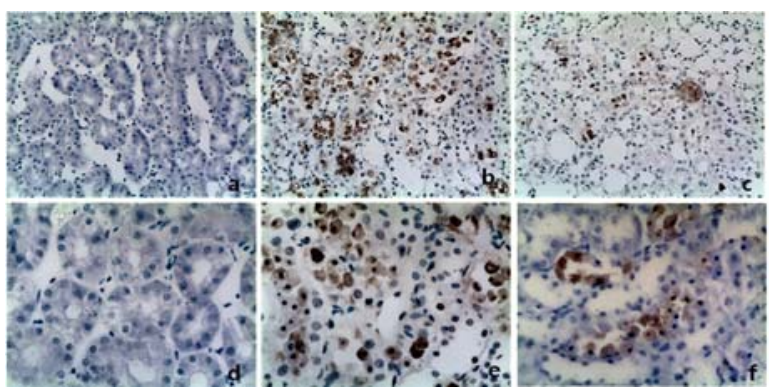

(B)

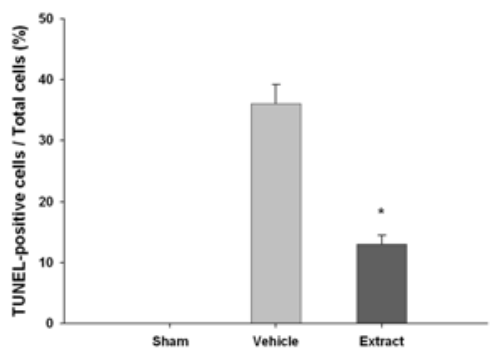

(C)

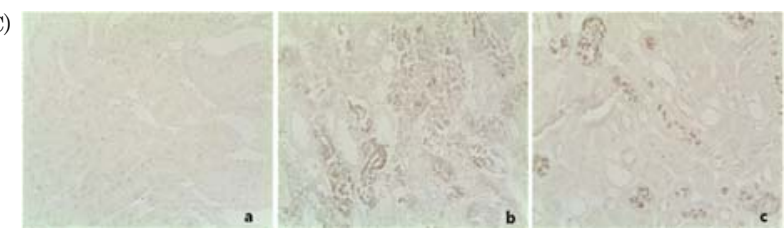

(D)

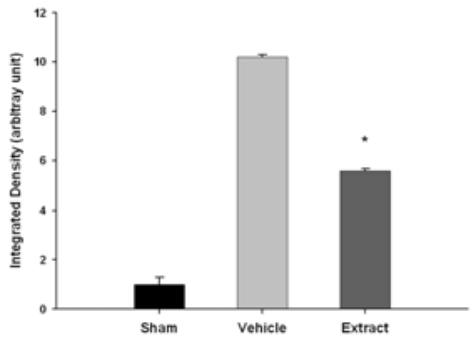

Fig. 3. Assessment of apoptotic cells by TUNE staining and assessment of caspase-3 activation by immunohistochemistryin a rat model of ischemia/reperfusion injury. A. Representative photomicrographs of the renal tissue sections stained with TUNEL at two different magnifications were presented: (a) \& (d) sham $(n=5)$ at $200 x$ and $400 x$, respectively, (b) \& (e) vehicle-treated $(n=7)$ at $200 x$ and $400 x$, respectively, and (c) \& (f) extract-treated group $(n=7)$, at 200x and 400x, respectively. B. Quantitative assessment of the TUNEL-positive cells was presented with TUNEL-positive cells/total cells ratio. Each column represents the means \pm SEM. ${ }^{*} P<0.05$ vs. vehicle-treated group. C. Representative photomicrographs of the renal tissue sections stained with immunohistochemistry for activated caspase-3 at magnification 400x were presented: (a) sham $(n=5)$, (b) vehicle-treated $(n=7)$, and (c) extract-treated group $(n=7)$, respectively. D. Quantitative assessment of activated caspase-3 produced from cleavage of procaspase-3 was presented, with the value for the sham group arbitrarily set as 1.0. Each column represents the means \pm SEM. ${ }^{*} P<0.05$ vs. vehicle-treated group.

\section{Association of $\mathrm{Bcl}-2$ and Bax in mediating} antiapoptotic effect of the extract
The expressed levels of Bcl-2 and Bax, which are antiapoptotic and proapoptotic proteins, respectively, were evaluated by immunohistochemistry as Bax/Bcl-2 ratio is the key indicator of apoptotic effect ${ }^{9)}$ (Fig. 4). Representative photomicrographs revealed that color intensities of Bcl-2 and Bax in the vehicle-treated group $(n=7)$ were markedly lower and higher, respectively, than those in the sham group $(n=5)$, whereas color intensities of Bcl-2 and Bax in the extract-treated group $(n=7)$ were markedly higher and lower, respectively, than those in the vehicle-treated group (Fig. 4A). The $\mathrm{Bax} / \mathrm{Bcl}-2$ ratios were used to quantify these findings (Fig. 4B). The ratio was significantly lower in the extract-treated group $(n=7)$ compared to that in the vehicle-treated group $(\mathrm{n}=7)(1.5 \pm 0.1$ versus $2.5 \pm 0.4$, $P<0.05)$, whereas that in the sham group $(\mathrm{n}=5)$ remained relatively low $(1.0 \pm 0.3)$.

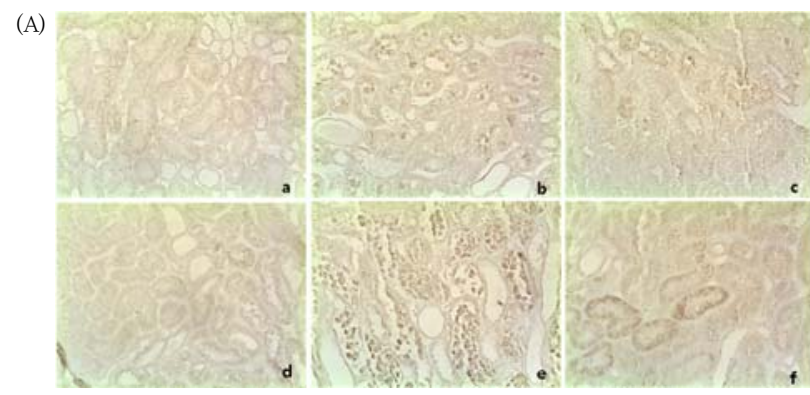

(B)

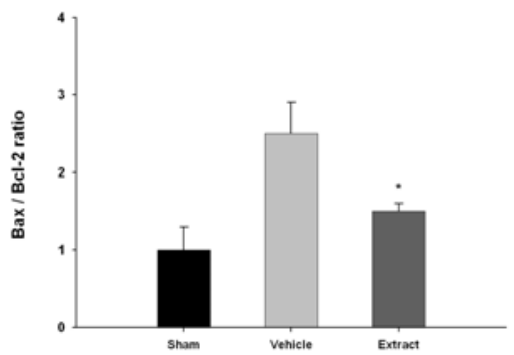

Fig. 4. Assessment of Bcl-2 and Bax expression by immunohistochemistry in a rat model of ischemia/reperfusion injury. A. Representative photomicrographs of renal tissue sections stained with immunohistochemistry for Bcl-2 and Bax at magnification 400x were presented: (a), (b), and (c); Bcl-2 for sham $(n=5)$, vehicle-treated $(n=7)$, and extract-treated group $(n=7)$, respectively, (d), (e), and (f); Bax for sham $(n=5)$, vehicle-treated $(n=7)$, and extract-treated group $(n=7)$, respectively. B. Quantitative assessment of $\mathrm{Bax} / \mathrm{Bcl}-2$ ratio was presented. Each column represents the means \pm S.E. ${ }^{*} P<0.05$ vs. vehicle-treated group.

\section{Discussion}

In this study, we demonstrated that the methanol extract of Cassia mimosoides var. nomame Makino (CM) protected the rat kidneys against ischemia/reperfusion injury by attenuating apoptosis when administered through an intraperitoneal injection prior to the induction 
of ischemia.

As previously noted, occlusion of the renal artery leads the kidney to an anoxic state. The consequent depletion of ATP triggers a cascade of events in the cells, and, eventually, result in the cell death by apoptosis or necrosis, depending on the severity and duration of ATP depletion ${ }^{7,9,20)}$. Furthermore, restoration of the blood flow exacerbates the situation by generating more reactive oxygen species (ROS) which further damage cellular structures such as membranes and proteins ${ }^{21)}$. Consequently, ROS augments cell death initiated by ischemia. The cells and cell debris detached from the tubules block the tubules located further downstream. The blockage allows wastes like creatinine in the tubular fluid to leak back into the peritubular capillaries ${ }^{3,6)}$. These events contribute to the reduction of effective glomerulus filtration rate (GFR), resulting in the elevation of serum creatinine level in the blood, which is a classical biomarker in assessing renal function ${ }^{22)}$.

Considering the aforementioned cascade of events occurring in renal ischemia/reperfusion, the findings of this study clearly demonstrated that the extract attenuated damage and subsequent death of the tubular epithelial cells in the outer medulla region as revealed by H\&E staining. Other studies using a similar rat or mouse model have also noted that the outer medulla region was especially susceptible to ischemia/reperfusion injury when the extent or damage was graded on score systems ${ }^{19,23)}$.

We have demonstrated using $\mathrm{DPPH}$, acting as a radical donor in in vitro system ${ }^{24)}$, that the extract has the same radical scavenging activity as ascorbic acid, a strong antioxidant, only at twice as high a concentration. As ascorbic acid reduced acute kidney injury in a rat model of ischemia/reperfusion ${ }^{25)}$, attenuation of the damage by the extract might also be associated with removal of ROS mediated by radical scavenging activity of the extract.

We have also shown that the ability of the extract to suppress the generation of ROS contributed to the attenuation of apoptosis by blocking the cascade of apoptotic pathways as shown by the reduction of Bax/Bcl-2 ratio, inhibition of caspase-3 activation, and consequent attenuation in the generation of DNA nicks. Assessment of apoptosis by measuring caspase-3 activation and generation of DNA nicks with immunohistochemistry and TUNEL staining, respectively, has been applied to rats previously ${ }^{9,26)}$. Finally, it was observed that the extract reduced tubular epithelial cell death by apoptosis, together with necrosis. The reduction consequently contributed to the improvement of renal function as assessed by reduction of serum creatinine level. In agreement with the findings of this study, overexpression of Bcl-2 protein in tubular cells in vitro and in vivo by viral vector-mediated gene transfer triggered inhibition of caspase -3 expression and attenuation of apoptosis under ischemia/reperfusion conditions ${ }^{27)}$. This finally accompanied inhibition of elevation of serum creatinine level, which conferred CM a renoprotective property. It is not surprising to find plants like Cassia mimosoides var. nomame Makino, showing renoprotective qualities, because over 170 plant species have already been reported to have such properties $^{28)}$. In addition, formulas containing a mixture of constituents prepared from several plants have also been successful in treating renal diseases ${ }^{29)}$. In this regard, Cassia mimosoides var. nomame Makino can also be a candidate for a renoprotective agent. This is particularly relevant as a method for large scale cultivation of CM has already been developed in Korea, rendering it feasible to get a large amount of the extract ${ }^{30)}$. In the future, it will be also possible to identify active compounds contributing to renal protection in $\mathrm{CM}$ because the ethyl acetate fraction ${ }^{14)}$, an active fraction attenuating cerebral ischemia/reperfusion injury, and some chemical components of CM like daucosterol and flavan have already been identified, at least in part $^{12,13)}$

\section{Conclusions}

In this study we revealed novel activities of Cassia mimosoides var. nomame Makino in attenuating renal injury:

1. A methanol extract of Cassia mimosoides var. nomame Makino has a radical scavenging activity in in vitro studies

2. The extract reduced tubular epithelial cell injury by blocking apoptotic cascades in rats.

3. The extract lowered serum creatinine concentration in rats.

In summary, this study showed that prophylactic administration of Cassia mimosoides var. nomame Makino was able to prevent ischemic acute kidney injury by removing ROS generated during ischemia/reperfusion and inhibiting apoptosis of tubular epithelial cells. Thus, the extract can be developed as a prophylactic agent to prevent acute kidney injury in clinical settings where acute kidney injury is expected to occur, such as post-surgery in heart. 


\section{Acknowledgement}

This research was supported by High Value-added Food Technology Development Program, Ministry of Agriculture, Food and Rural Affairs.

\section{References}

1. Lopes JA, Jorge S. The RIFLE and AKIN classifications for acute kidney injury: a critical and comprehensive review. Clin Kidney J. 2013 ; 6 : 8-14.

2. Chen YP. Definition and classification of acute kidney injury: contributions and problems in the clinical practice. Chin J Integr Med. 2010 ; 16 : 204-6.

3. Abuelo JG. Normotensive ischemic acute renal failure. N Engl J Med. 2007 ; 357 : 797-805.

4. Venkataraman R, Kellum JA. Defining acute renal failure: the RIFLE criteria. J Intensive Care Med. $2007 ; 22: 187-93$.

5. Bonventre JV, Yang L. Cellular pathophysiology of ischemic acute kidney injury. J Clin Invest. 2011 ; 121 : 4210-21.

6. Devarajan P. Update on mechanisms of ischemic acute kidney injury. J Am Soc Nephrol. 2006 ; 17 : 1503-20.

7. Sabbahy ME, Vaidya VS. Ischemic kidney injury and mechanisms of tissue repair. Wiley Interdiscip Rev Syst Biol Med. 2011 ; 3 : 606-18.

8. Rodriguez F, Bonacasa B, Fenoy FJ, Salom MG. Reactive oxygen species in the renal ischemia/reperfusion injury. Curr Pharm Des. 2013 ; 19 : 2776-94.

9. Havasi A, Borkan SC. Apoptosis and acute kidney injury. Kid Int. $2011 ; 80$ : 29-40.

10. Baek HS, Lim SH, Ahn KS, Lee JW. Hot water extract of Triticum aestivum L. (common wheat) ameliorates renal injury by inhibiting apoptosis in a rat model of ischemia/reperfusion. Kor $\mathrm{J}$ Herbology. $2013 ; 28: 7-15$.

11. Yamamoto M, Shimura S, Itoh T, Ohsaka T, Egawa M, Inoue S. Anti-obesity effects of lipase inhibitor CT-II, an extract from edible herbs, Nomame Herba, on rats fed a high-fat diet. Int J Obes Relat Metab Disord. 2000 ; 24 : 758-64.

12. Park JH, Kwon SJ. Isolation of daucosterol and napthlene glucoside from seeds of Cassia mimosoides var. nomame Makino. Kor J Plant Res. 2009 ; 22 : $26-30$

13. Hatano $\mathrm{T}$, Yamashita $\mathrm{A}$, Hashimoto $\mathrm{T}$, Ito $\mathrm{H}$, Kubo N, Yoshiyama M, Shimura S, Itoh Y, Okuda T, Yoshida T.Flavan dimers with lipase inhibitory activity from Cassia nomame. Phytochem.1997 ; 46 : 893-900.
14. Kim KH, Lee JW. Methanol extract of Cassia mimosoides var. nomame and its ethyl acetate fraction attenuate brain damage by inhibition of apoptosis in a rat model of ischemia-reperfusion. J Food Sci Nutr. $2010 ; 15: 255-61$.

15. Lim SH, Lee JW. Methanol extract of Cassia mimosoides var. nomame attenuates myocardial injury by inhibition of apoptosis in a rat model of ischemia-reperfusion. Prev Nutr Food Sci. 2012 ; 17 : 177-83.

16. Eltzschig HK, Eckle T. Ischemia and reperfusion-from mechanism to translation. Nat Med. 2011 ; 17 : 1391-401.

17. Jung HJ, Kim MJ, Lim SH, Park JH, Lee HK, Ahn KS, JW Lee. The effect of extract of Paeonia lactiflora on the improvement of ischemic acute renal failure. Korean J Nephrol. 2009 ; 28 : 180-9.

18. Kuriakose GC, Kurup MG. Effects of Aulosira fertilisima against cisplatin-induced nephrotoxicity and oxidative stress in rats. Ren Fail. 2010 ; 32 : 224-33.

19. Kelly KJ, Williams WW Jr, Colvin RB, Meehan SM, Springer TA, Gutierrez-Ramos JC, Bonventre $\mathrm{JV}$. Intracellular adhesion molecule-1-deficient mice are protected against ischemic renal injury. J Clin Invest. 1996 ; 97 : 1056-63.

20. Heyman SN, Rosenberger C, Rosen S. Experimental ischemia-reperfusion: biases and myths-the proximal vs. distal hypoxic tubular injury debate revisited. Kidney Int. 2010 ; 77 : 9-16.

21. Erdogan H, Fadillioglu E, Yagmurca M, Ucar M, Irmak MK. Protein oxidation and lipid peroxidation after renal ischemia-reperfusion injury: protective effects of erdosteine and $\mathrm{N}$-acetylcysteine. Urol Res. 2006 ; 34 : 41-6.

22. Schiffl H, Lang SM. Update on biomarkers of acute kidney injury: moving closer to clinical impact? Mol Diagn Ther. 2012 ; 16 : 199-207.

23. Wei Q, Dong Z. Mouse model of ischemic acute kidney injury: technical notes and tricks. Am J Physiol Renal Physiol. 2012 ; 303 : F1487-94.

24. Kedare SB, Singh RP. Genesis and development of DPPH method of antioxidant assay. J Food Sci Technol. $2011 ; 48 ： 412-22$.

25. Korkmaz A, Kolankaya D. The protective effects of ascorbic acid against renal ischemia-reperfusion injury in male rats. Ren Fail. 2009 ; 31 : 36-43.

26. Wagner M, Cadetg P, Ruf R, Mazzuchelli L, Ferrari P, Redaelli CA. Heme oxygenase-1 attenuates ischemia/reperfusion-induced apoptosis and improves survival in rat renal allografts. Kidney Int. 2003 ; $63: 1564-73$.

27. Chien CT, Chang TC, Tsai CY, Shyue SK, Lai MK. Adenovirus-mediated bcl-2 gene transfer 
inhibits renal ischemia/reperfusion induced tubular oxidative stress and apoptosis. Am J Transplant. $2005 ; 5$ : 1194-203.

28. Lien EJC, Lien LLM, Wang R, Wang J. Phytochemical analysis of medicinal plants with kidney protective activities. Chin J Integr Med. 2012 ; 18 : 790-800.

29. Zheng XY, Wei RB, Shi SZ, Yin Z, Chen XM. Effects of Fufang Shenhua tablet on the expression of Toll-like receptors during acute kidney injury induced by ischemia-reperfusion in rats. Chin $\mathrm{J}$ Integr Med. 2012 ; 18 : 928-924.

30. Cho NK, Kang YK, Song CK, Oh EK, Cho YI. Effects of phosphate application rate on growth, yield and chemical composition of Cassia mimosoides var. nomame. Korean J Crop Sci. 2000 ; 45 : 163-66. 